\title{
A REMARK ON WARING DECOMPOSITIONS OF SOME SPECIAL PLANE QUARTICS*
}

\author{
ALESSANDRO DE PARIS ${ }^{\dagger}$
}

\begin{abstract}
Motivated by questions on tensor rank, this work concerns the following unexpected result concerning Waring decompositions of plane quartics containing a double line, along with some preparatory and additional remarks. Let $x, l_{1}, \ldots, l_{7}$ be linear forms and $q$ a quadratic form on a vector space of dimension 3 . If $x^{2} q=l_{1}^{4}+\cdots+l_{7}^{4}$ and the lines $l_{1}=0, \ldots, l_{7}=0$ in $\mathbb{P}^{2}$ intersect $x=0$ at seven distinct points, then the line $x=0$ is (possibly improperly) tangent to the conic $q=0$.
\end{abstract}

Key words. Waring decomposition, Tensor rank, Secant varieties.

AMS subject classifications. 15A99, 14M99.

1. Introduction. Let $f \in K\left[x_{0}, \ldots, x_{n}\right]$ be a degree $d$ homogeneous polynomial and

$$
f=l_{1}^{d}+\cdots+l_{r}^{d}
$$

be a decomposition of $f$ as a sum of $d$-th powers of linear forms. The minimum number of summands for such a decomposition is called the Waring rank of $f$, or the symmetric tensor rank (the latter term is sometimes used with a different meaning: see [11, where the Waring rank is called polar rank instead). We shall refer to it simply as the rank of $f$. By a Waring decomposition, we mean a decomposition (1.1) that is minimal, i.e., with $r$ equal to the rank.

The problem of finding the maximum possible rank, given $d$ and $n$, is called the little Waring problem for polynomials in 6 . We refer the reader to $[\underline{6}$ for a friendly exposition and to 9 for an extensive and up-to-date survey on the role of tensor rank theory in a broad range of applications. The latter will also be our default reference for basic terminology.

When $n=1$, the answer is known: a detailed description is given by the ComasSeiguer theorem (see [5] or, e.g., [9, Theorem 9.2.2.1]). In the case $(d, n)=(3,2)$, the maximal rank is five (see [10, Section 8]). The answer for $(d, n)=(4,2)$, seven,

\footnotetext{
* Received by the editors on December 27, 2012. Accepted for publication on July 8, 2013. Handling Editor: Bryan L. Shader.

†Dipartimento di Matematica e Applicazioni "R. Caccioppoli", Università degli Studi di Napoli Federico II, Via Cintia, Monte S. Angelo, I-80126 Napoli, Italy (deparis@unina.it).
} 
was given in [8] and, surprisingly, it seems not to be widely known. For instance, there is no mention of it in [9], in particular in the preamble to Chapter 9. But, beyond the Waring problem, much work has recently been done to understand the rank stratification of polynomials. A careful account in the case $(d, n)=(4,2)$ is given by [2, Theorem 44] (cf. also [9, Theorem 10.9.3.2]), among a considerable number of other interesting results. Further developments in [1] give the rank stratification for polynomials of border rank four, for all $(d, n)$, yielding in particular a positive answer in the case $n=3$ to a conjecture by J. H. Rhodes about cubic polynomials.

According to [9, 0.2, p. xv], 'Results [...] indicate there is beautiful geometry associated to rank that is only beginning to be discovered.'. Several recent papers, and the book [9] itself, give evidence that classical, even elementary, results from algebraic geometry are very useful in tensor rank questions, and a bit more advanced tools such as nonreduced schemes have considerably increased the knowledge. We may likely expect that deeper tools from algebraic geometry, e.g., the homological ones, will provide a deeper understanding when carefully adapted to the subject. In our opinion, outstanding results in this respect are some of those in [9, Part 4], and [3. Proposition 3.1] (to compare its scope and simplicity with what was previously known, see [9, 3.2.3 and 9.3.3]).

We believe that, in such situations, even results that look very partial and far from being outstanding may positively contribute to the discovery mentioned in 9 , loc. cit.]. For instance, quartics that are decomposed in a double line and a conic seem to yield matter for good tests (think, e.g., of plane cubics, for which the maximal rank is achieved by a conic together with a line tangent to it). We also mention that such quartics are among the simplest examples of what in [7] are called padded polynomials. That is why the focus of the present work is on Proposition 3.2. It was computationally detected, it looks rather unexpected, and the proof required some considerations that are not completely routine in this context. A few additional remarks and applications, though of some independent interest, are presented in view of that result.

We have tried to optimize the technical background at the beginning of Section 3 , so that arguments in the subsequent proof could be developed both concisely and in detail. To prevent the 'optimized language' from obscuring some more elementary facts, in Section 2 we anticipated some results in an elementary setting (at the cost of a lesser optimization in the arguments). Proposition 2.2 in that preliminary section deals with quartics that are decomposed in a double line and a nondegenerate conic not tangent to the line. In spite of its ancillary illustrative role, it has its own interest, because the proof includes a quite detailed description of the space of Waring decompositions of such quartics and gives quite a direct check that their rank is six (an earlier reference for this fact is [2, Case (2) in the proof of Theorem 44], but we found the indirect argument there rather obscure). 
2. Preliminary remarks. First of all,

we fix an algebraically closed field $K$ of zero characteristic

(generally one may assume $K=\mathbb{C}$ : see, e.g., [9, Subsection 1.2.1]). That is a common assumption when one deals with the rank of polynomials, possibly because positive characteristics often lead to cumbersome subtleties. Moreover, note that $x y$, as a polynomial over a field of characteristic 2 , cannot be expressed as a sum of the squares of linear forms; similarly for $-x^{2}$ as a polynomial over $\mathbb{R}$. However, we shall outline how things go for arbitrary fields by making a quick separate remark.

We start with some very elementary considerations in the usual coordinate settings. Let us consider six linear forms in a polynomial ring $K\left[x_{0}, x_{1}, x_{2}\right]$ :

$$
l_{1}:=x_{0}+h_{1} x_{1}+k_{1} x_{2}, \ldots, l_{6}:=x_{0}+h_{6} x_{1}+k_{6} x_{2} .
$$

We seek a linear combination of $l_{1}^{4}, \ldots, l_{6}^{4}$ such that the quartic curve it represents doubly contains the line $x_{2}=0$ :

$$
\alpha_{1} l_{1}^{4}+\cdots+\alpha_{6} l_{6}^{4}=x_{2}^{2} q\left(x_{0}, x_{1}, x_{2}\right)
$$

with $q$ being a quadratic form. Simple arguments indicate that, for a generic choice of $l_{1}, \ldots, l_{6}$, one does not expect such a combination to exist. In geometric terms, we are dealing with the intersection of an osculating 5 -space to the 4-th Veronese surface $S \subset \mathbb{P}^{14}$ and a secant 5-space. On the other hand, one expects plenty of special choices of $l_{1}, \ldots, l_{6}$ leading to (2.2). Note also that the generic rank for ternary quartics is expected to be 5 , but it is actually 6 (in fact, $(d, n+1)=(4,3)$ is an exceptional case of the Alexander-Hirschowitz theorem; see [9, Theorem 3.2.2.4]).

The expectation of many solutions for (2.2) could be threatened by the existence of special quartics of higher rank than the generic. More precisely, if the conic represented by $q$ is nondegenerate and tangent to the line $x_{2}=0$, then the rank of $x_{2}^{2} q$ is seven (see [9, Theorem 10.9.2.1] or [2, case (1) in the proof of Theorem 44, (p. 50)]). If this were the case for all quadratic forms $q \neq 0$, then (2.2) would admit no solutions, apart from those with $q=0$. Now, trivially, when $q=x_{2}^{2}$, the rank of $x_{2}^{2} q$ is one. Even if $q=x_{2} l$, with $l$ a linear form not proportional to $x_{2}$, then $q x_{2}^{2}$ is of rank 4 (see, e.g., 5]). Incidentally, in such cases we do not immediately get a solution for (2.2), because $l_{1}, \ldots, l_{6}$, though generic, are in the form (2.1).

When $q=0$ has no singular points lying on $x_{2}=0$, a good lower bound on the rank is given by [10, Theorem 1.3] (also reported in [9, 9.2.1.4]). In this case we have, in notation of that theorem, $\operatorname{dim} \Sigma_{1}=1$ and $\operatorname{rank} \phi_{1,3}=3$; this way we get that the rank is at least five. The same bound may be deduced by taking $s=2$ in the same theorem, i.e., by looking at $\Sigma_{2}$ and $\phi_{2,2}$ (with a bit extra work to detect the rank of 
$\left.\phi_{2,2}\right)$. Note also that assuming $s=2$ in [10, Section 6, proof of Theorem 1.3], we get a geometric picture which has some interesting relationships with the results we are going to prove in this paper.

A solution for (2.2) is given by the following example.

ExAmPle 2.1. Set

$$
\begin{array}{cl}
l_{1}:=x_{0}, \quad l_{2}:=x_{0}+x_{2}, & l_{3}:=x_{0}-x_{2}, \\
l_{4}:=x_{0}+x_{1}, \quad l_{5}:=x_{0}+x_{1}+x_{2}, & l_{6}:=x_{0}+x_{1}-x_{2} .
\end{array}
$$

We have

$$
2 l_{1}^{4}-l_{2}^{4}-l_{3}^{4}+2 l_{4}^{4}-l_{5}^{4}-l_{6}^{4}=-4\left(6 x_{0}^{2}+6 x_{0} x_{1}+3 x_{1}^{2}+x_{2}^{2}\right) x_{2}^{2}
$$

and $6 x_{0}^{2}+6 x_{0} x_{1}+3 x_{1}^{2}+x_{2}^{2}=0$ is nondegenerate and not tangent to $x_{2}=0$.

The above decomposition looks somewhat special, because the set of $h_{i}$ 's reduces to only two values. That is why we find it a bit surprising that this is a necessary condition, as we quickly explain now.

Proposition 2.2. In the above notation, when the conic $q=0$ is nondegenerate and not tangent to the line $x_{2}=0$, for every decomposition (2.2), the $\alpha_{i}$ 's are all nonzero and the six $h_{i}$ 's reduce to only two values, with correspondence three-to-one.

Proof. Let us split $l_{i}=L_{i}+k_{i} x_{2}$, with $L_{i}:=x_{0}+h_{i} x_{1}$, think of $L_{1}, \ldots, L_{6}$ as fixed and look for appropriate values of $\alpha_{1}, \ldots, \alpha_{6}, k_{1}, \ldots, k_{6}$. We find ourselves dealing with a system of equations

$$
\begin{aligned}
& \sum_{i=1}^{6} \alpha_{i} h_{i}^{d}=0,0 \leq d \leq 4, \\
& \sum_{i=1}^{6} \alpha_{i} k_{i} h_{i}^{d}=0,0 \leq d \leq 3 .
\end{aligned}
$$

Suppose that $h_{1}, \ldots, h_{6}$ are distinct. Equations (2.3) involve some Vandermonde determinants, so that we have a solution $\alpha=\left(\alpha_{1}, \ldots, \alpha_{6}\right)$, with all nonzero entries $\alpha_{i}$, which is unique up to a scalar factor. The subsystem with $0 \leq d \leq 3$ admits a twodimensional space of solutions $\beta=\left(\beta_{1}, \ldots, \beta_{6}\right)$. Solutions $k=\left(k_{1}, \ldots, k_{6}\right)$ of (2.4) are given by $k_{i}=\beta_{i} / \alpha_{i}$, and therefore they form a two-dimensional space. But since

$$
\sum_{i=1}^{6} \alpha_{i} L_{i}\left(x_{0}, x_{1}\right)^{4}=0
$$


setting $l_{i}=L_{i}\left(x_{0}+\varrho_{0} x_{2}, x_{1}+\varrho_{1} x_{2}\right)$ for arbitrary $\rho_{0}, \rho_{1} \in K$, we get that (2.2) holds with $q=0$. Hence, the whole two-dimensional space of solutions $k$ for (2.4) must give $q=0$. This shows that no nonzero quartics of type $x_{2}^{2} q$ can arise when the $h_{i}$ 's are distinct.

Suppose then that $h_{1}, \ldots, h_{6}$ are not distinct. Let us subdivide the six $l_{i}$ 's in groups, according to the distinct values of the $h_{i}$ 's, and call $f_{1}, \ldots, f_{s}(s \leq 5)$ the corresponding linear combinations of their fourth powers, with the coefficients being the $\alpha_{i}$ 's. Since fewer than six distinct $L_{i}^{4}$ 's are linearly independent, in view of (2.5) we have that each $f_{j}$ contains $x_{2}$ as a factor, and it vanishes if it consists of only one term as a linear combination $\alpha_{i} l_{i}^{4}$ (simply because $\alpha_{i}$ must vanish). Therefore, dividing each nonzero $f_{j}$ by $x_{2}$, we get $g_{1}, \ldots, g_{t}$ such that

$$
x_{2} q=g_{1}+\cdots+g_{t}
$$

and $1 \leq t \leq 3$. Each $g_{j}$ must represent a cone (union of lines) with vertex $V_{j}$ of the form $\left(h_{i},-1,0\right)$ for some $i$ depending on $j$. Moreover, each cone $g_{j}=0$ either contains the line $x_{2}=0$, or intersects it into $3 V_{j}$. Since the $V_{j}$ 's are distinct, in view of (2.6) we deduce that each $g_{j}$ contains $x_{2}$ as a factor. But when $f_{j}$ is a linear combination of only two of the $l_{i}$ 's, then it cannot contain $x_{2}^{2}$ as a factor (from an algebro-geometric viewpoint, this is an elementary fact about linear series on a line; it may also be deduced from [9, Theorem 9.2.1.4]). This proves that $t \leq 2$. On the other hand, it cannot be $t=1$ because $q$ does not represent a cone. Moreover, each of the two nonzero $f_{j}$ 's must be a linear combination of three (and not less) of the $l_{i}^{4}$ 's, and this immediately leads to the statement.

Following from the above line of thought, we turn our attention to a quartic $x_{2}^{2} q$, with $q$ representing a nondegenerate conic tangent to the line $x_{2}=0$. As mentioned before, it is known that such a quartic is of rank seven. By some trial calculations, one can find $\alpha_{1}, \ldots, \alpha_{7}$ and $l_{1}, \ldots, l_{7}$ such that $\alpha_{1} l_{1}^{4}+\cdots+\alpha_{7} l_{7}^{4}$ is a quartic of that type, where $l_{7}=x_{0}+h_{7} x_{1}+k_{7} x_{2}$, similarly to the preceding $l_{1}, \ldots, l_{6}$.

What is new in this case is that $h_{1}, \ldots, h_{7}$ can be distinct. In our opinion, it would be reasonable to expect that for a generic linear combination of $l_{1}^{4}, \ldots, l_{7}^{4}$ that yields $x_{2}^{2} q$, the quadric $q=0$ should be not tangent to the line $x_{2}=0$ (i.e., the tangency case should be special). The main result we are going to prove asserts that this expectation fails.

Before going into the proof, we want to briefly discuss some computational aspects. Put into elementary terms as before, our result reduces to the following assertion. If the $h_{i}$ 's are distinct, and $k_{i}, \alpha_{i}$ such that

$$
\sum_{i=1}^{7} \alpha_{i} h_{i}^{d}=0, \quad 0 \leq d \leq 4, \quad \sum_{i=1}^{7} \alpha_{i} k_{i} h_{i}^{d}=0, \quad 0 \leq d \leq 3,
$$


then we have

$$
\left(\sum_{i=1}^{7} \alpha_{i} k_{i}^{2} h_{i}\right)^{2}-\left(\sum_{i=1}^{7} \alpha_{i} k_{i}^{2}\right)\left(\sum_{i=1}^{7} \alpha_{i} k_{i}^{2} h_{i}^{2}\right)=0
$$

In principle, this can be proved by a brute force calculation. Indeed, let $f$ be the above polynomial in $h_{i}, k_{i}, \alpha_{i}$; let $g:=\prod_{j>i}\left(h_{i}-h_{j}\right)$ and $\mathfrak{a}$ the ideal generated by all polynomials in (2.7). Then it would suffice to check that $f g \in \sqrt{\mathfrak{a}}$. We have tried to perform this check with CoCoA (see [4]) on a common computer. But even with the simpler (sufficient) condition $f g \in \mathfrak{a}$, and even with some of the indeterminates specialized, the calculation was out of reach. Only some tests with many specializations ended up (with a positive answer).

3. Main result. The symmetric algebra $S^{\bullet} V$ of a $K$-vector space $V$ will be denoted by $S_{V}$. The projective space $\mathbb{P} V$ will simply be the set of proportionality classes of nonzero vectors in $V$. An $f \in S_{V^{*}}$ will be interpreted, as usual, as a polynomial function on $V$. Dually, elements of $S_{V}$ are interpreted as polynomial functions on $V^{*}$, and we find it comfortable to denote the value of $s \in S_{V}$ on $x \in V^{*}$ by

$$
x(s)
$$

(for instance, with $v \in V$, we allow ourselves to say that $x\left(v^{n}\right)=x(v)^{n}=x^{n}(v)$ ).

To speed up calculations, we assume the following reasonable conventions. When dealing with $n$-tuples of polynomials, say $f=\left(f_{1}, \ldots, f_{n}\right), g=\left(g_{1}, \ldots, g_{n}\right)$, we multiply them by the rule

$$
f g=\left(f_{1} g_{1}, \ldots, f_{n} g_{n}\right)
$$

(Hadamard product): It is nothing but the multiplication in the ordinary cartesian product ring $\left(S_{V^{*}}\right)^{n}$ (or $\left.\left(S_{V}\right)^{n}\right)$. We shall also make use of the standard bilinear form $\left(S_{V^{*}}\right)^{n} \times\left(S_{V^{*}}\right)^{n} \rightarrow S_{V^{*}}:$

$$
f \cdot g=f_{1} g_{1}+\cdots+f_{n} g_{n}
$$

for which we shall keep the dot notation. Note that $f g \cdot h=f \cdot g h$. These operations can be performed, in particular, on elements of $K^{n} \subseteq\left(S_{V^{*}}\right)^{n}$. Since we are considering $K^{n}$ also as a ring, sometimes 1 will stand for the identity element in this ring, i.e., $(1, \ldots, 1)$. This way, a decomposition (1.1) of $f \in S^{d} V^{*}$ may be written

$$
f=1 \cdot l^{d}
$$

with $l \in\left(V^{*}\right)^{n} \subset\left(S_{V^{*}}\right)^{n}$ being an $n$-tuple of linear forms. 
Let us also recall the notation $\bar{f}$ for the polarization of $f \in S^{d} V^{*}$, i.e., the symmetric $d$-multilinear form such that

$$
f(v)=\bar{f}(v, \ldots, v)
$$

$\left(d ! \bar{f}\right.$ may be regarded as the image of $f$ through a canonical map $\left.S^{d} V^{*} \rightarrow\left(S^{d} V\right)^{*}\right)$. Moreover, partial polarizations of $f$,

$$
f_{\delta, d-\delta}: S^{\delta} V \rightarrow S^{d-\delta} V^{*}
$$

are also defined (see [9, 2.6.6]; cf. also [2, Definition 17]).

REMARK 3.1. Let $W$ be a $K$-vector space and $\ell \in W^{*}$. The partial polarization

$$
\ell_{d, \delta}^{d+\delta}: S^{d} W \rightarrow S^{\delta} W^{*}
$$

of $\ell^{d+\delta} \in S^{d+\delta} W^{*}$ acts by associating each $t \in S^{d} W$ with the linear form

$$
S^{\delta} W \rightarrow K, \quad s \mapsto \ell(t s)=\ell(t) \ell(s) .
$$

Since partial polarization $f \mapsto f_{d, \delta}$ is a $K$-linear procedure, if $L \in\left(W^{*}\right)^{n}$ is an $n$-tuple of linear forms, then for all $a \in K^{n}$, the partial polarization $S^{d} W \rightarrow S^{\delta} W^{*}$ of $a \cdot L^{d+\delta}$ acts as follows:

$$
s \mapsto a \cdot L(t) L(s)=a L(t) \cdot L(s), \quad \forall t \in S^{d} W .
$$

Proposition 3.2. Let $V$ be a $K$-vector space, $\operatorname{dim} V=3$, and let $x \in V^{*}$, $q \in S^{2} V^{*}, x, q \neq 0$. If

$$
x^{2} q=l_{1}^{4}+\cdots+l_{7}^{4}
$$

with $l_{1}, \ldots, l_{7} \in V^{*}$ such that the lines $l_{1}=0, \ldots, l_{7}=0$ in $\mathbb{P} V$ intersect $x=0$ at seven distinct points, then the line $x=0$ is (possibly improperly) tangent to the conic $q=0$.

Proof. Let $W:=x^{\perp}=\operatorname{ker} x \subseteq V$ and let us use capital letters for restrictions to $W$ :

$$
L_{1}:=l_{1} \uparrow_{W}, \ldots, L_{7}:=l_{7} \uparrow_{W} .
$$

Set also $l:=\left(l_{1}, \ldots, l_{7}\right) \in\left(V^{*}\right)^{7} \subset\left(S_{V^{*}}\right)^{7}$ and, similarly, $L:=\left(L_{1}, \ldots, L_{7}\right) \in\left(S_{W^{*}}\right)^{7}$. By the hypothesis on the intersections with $\mathbb{P} W: x=0$ we have that $\left[L_{1}\right], \ldots,\left[L_{7}\right]$ are distinct in $\mathbb{P} W^{*}$. This easily implies that $L_{1}^{d}, \ldots, L_{7}^{d}$ span $S^{d} W^{*}$ when $d \leq 6$, so that in this case the linear map

$$
\begin{aligned}
\varphi_{d}: K^{7} & \rightarrow S^{d} W^{*} \\
a & \mapsto a \cdot L^{d}
\end{aligned}
$$


is surjective. Therefore, we have

$$
\operatorname{dim} \operatorname{ker} \varphi_{d}=6-d, \quad d=0, \ldots, 6 .
$$

By Remark 3.1, and taking into account that $L\left(w^{d}\right)=L^{d}(w)$ for all $w \in W$, we have that if $a \in \operatorname{ker} \varphi_{d+\delta}$ and $t \in S^{\delta} W$ then $a L(t) \in \operatorname{ker} \varphi_{d}$. Thus, whenever $a \in \operatorname{ker} \varphi_{d+\delta}$, we can define a linear map

$$
\begin{aligned}
\psi_{a, \delta, d}: \quad S^{\delta} W & \rightarrow \operatorname{ker} \varphi_{d} \\
t & \mapsto a L(t)
\end{aligned}
$$

Again because of the hypothesis on the intersections, we have:

- a generator for $\operatorname{ker} \varphi_{5}$ is invertible in $K^{7}$ (i.e., all of its components are nonzero);

- with an invertible $a \in K^{7}$ and $\delta \leq 6$, the map $\psi_{a, \delta, d}$ is injective.

In particular, if $a$ is a generator for $\operatorname{ker} \varphi_{5}$ then the map $\psi_{a, 1,4}$ is injective. It is henceforth surjective, because $\operatorname{dim} W=\operatorname{dim} \operatorname{ker} \varphi_{4}=2$. But since $1 \cdot l^{4}=x^{2} q$, we have that $1 \in \operatorname{ker} \varphi_{4}$. This way we end up with a $w \in W$ such that

$$
a=\frac{1}{L(w)}
$$

generates $\operatorname{ker} \varphi_{5}$

Now let us pick $v \in V$ such that $x(v)=1$ and set $l_{v}:=l-l(v) x$, where

$$
l(v) x:=\left(l_{1}(v) x, \ldots, l_{7}(v) x\right) \in\left(V^{*}\right)^{7} \subset\left(S_{V^{*}}\right)^{7}
$$

(in other terms, we are writing to the right the $S_{V^{*}}$-module multiplication in $\left(S_{V^{*}}\right)^{7}$, to avoid ambiguities due to the evaluation at $v$ ). Clearly $l_{v}(v)=0$ and the restriction of $l_{v}$ to $W$ is again $L$. From

$$
1 \cdot\left(l_{v}+l(v) x\right)^{4}=1 \cdot l^{4}=x^{2} q
$$

we deduce that

$$
1 \cdot l_{v}^{4}+4\left(l(v) \cdot l_{v}^{3}\right) x \in x^{2} S^{2} V^{*} \subset S^{4} V^{*} .
$$

But from $1 \cdot L^{4}=0, l_{v}(v)=0$ easily follows $1 \cdot l_{v}^{4}=0$, hence $l(v) \cdot l_{v}^{3}$ is divisible by $x$. Therefore

$$
l(v) \cdot L^{3}=0
$$

that is, $l(v) \in \operatorname{ker} \varphi_{3}$. Since the injective linear map $\psi_{1 / L(w), 2,3}: S^{2} W \rightarrow \operatorname{ker} \varphi_{3}$ is also surjective by dimension reasons, we end up with a $b \in S^{2} W$ such that

$$
l(v)=\frac{L(b)}{L(w)} .
$$


With $Q:=q \uparrow_{W}$ and $\bar{Q}$ its polarization, (3.1) implies

$$
Q=6 l(v)^{2} \cdot L^{2}=6 \frac{L\left(b^{2}\right)}{L(w)^{2}} \cdot L^{2} .
$$

Therefore, for all $u \in W$ we have

$$
\bar{Q}(w, u)=6 \frac{L\left(b^{2}\right)}{L(w)} \cdot L(u)=0,
$$

since $L\left(b^{2}\right) / L(w)=\psi_{1 / L(w), 4,1}\left(b^{2}\right) \in \operatorname{ker} \varphi_{1}$. In other words, $w$ is in the kernel of the polarization $\bar{Q}$, which exactly means that $q=0$ and $x=0$ are tangent at $[w]$.

REMARK 3.3. In the above proof we did not use the fact that $K$ was assumed algebraically closed. For fields of characteristic $\geq 7$ the proof basically works without changes (one has only to be careful with definitions about symmetric powers and polarizations). In characteristic $5, \operatorname{ker} \varphi_{5}$ fails to be one-dimensional, but we can still find an invertible element in it. In characteristic 2 or 3 the result is rather trivial, because $q$ becomes divisible by $x$.

Though we have chosen quite a direct algebraic language, we prefer not to miss hinting at a more geometric interpretation, in view of possible generalizations. In notation of the proof of Proposition 3.2, let $Y:=\left\{\left[L_{1}\right], \ldots,\left[L_{7}\right]\right\} \subseteq \mathbb{P} W^{*}$. The maps $\varphi_{d}$ are dual to the restriction maps

$$
H^{0}(\mathcal{O}(d)) \rightarrow H^{0}\left(\mathcal{O}_{Y}(d)\right),
$$

and hence, their kernels are dual to $H^{1}\left(\mathcal{I}_{Y}(d)\right.$ ) (one might also note that they are naturally isomorphic to $\operatorname{Hom}\left(\mathcal{I}_{Y}(d), \omega\right)$, by Serre duality). The direct sum over $d$ of the $H^{1}\left(\mathcal{I}_{Y}(d)\right)$ 's is known as the Hartshorne-Rao module of $Y$, and the maps $\psi_{a, \delta, d}$ are byproducts of that module structure.

Acknowledgment. MIUR (Italian Ministry of Education, University and Research) provided partial financial support within a PRIN project, during the preparation of this work. We thank the referee for some interesting remarks.

Finally, the author warmly acknowledges Gennaro De Paris in the occasion of his $3^{4}$ th birthday, for his very early training support.

\section{REFERENCES}

[1] E. Ballico and A. Bernardi. Stratification of the fourth secant variety of Veronese varieties via the symmetric rank. Adv. Pure Appl. Math., 4:215-250, 2013.

[2] A. Bernardi, A. Gimigliano, and M. Idà. Computing symmetric rank for symmetric tensors. J. Symb. Comput., 46:34-53, 2011. 
[3] E. Carlini, M.V. Catalisano, and A.V. Geramita. The solution to the Waring problem for monomials and the sum of coprime monomials. J. Algebra, 370:5-14, 2012.

[4] CoCoA Team. CoCoA: a system for doing Computations in Commutative Algebra. Available at http://cocoa.dima.unige.it.

[5] G. Comas and M. Seiguer. On the rank of a binary form. Found. Comput. Math., 11:65-78, 2011.

[6] A.V. Geramita. Inverse systems of fat points: Waring's problem, secant varieties of Veronese varieties and parameter spaces for Gorenstein ideals. The Curves Seminar at Queen's, Vol. X, Queen's University, Kingston, 2-114, 1996.

[7] H. Kadish and J.M. Landsberg. Padded polynomials, their cousins, and geometric complexity theory. Preprint arXiv:1204.4693v1 [math.AG], 2012. Available at http://arxiv.org/abs/1204.4693.

[8] J. Kleppe. Representing a Homogenous Polynomial as a Sum of Powers of Linear Forms. Thesis for the degree of Candidatum Scientiarum, Department of Mathematics, University of Oslo, 1999.

[9] J.M. Landsberg. Tensors: Geometry and Applications. American Mathematical Society, Providence, RI, 2012.

[10] J.M. Landsberg and Z. Teitler. On the ranks and border ranks of symmetric tensors. Found. Comput. Math., 10:339-366, 2010.

[11] T.H. Pate. Lower bounds for polar and tensor ranks. Linear Multilinear Algebra, 21:277-287, 1987. 